\title{
TV/Series
}

12 | 2017

Littérature et séries télévisées/Literature and TV series

\section{Penny Dreadful ou comment la littérature peut-elle donner naissance à une série?}

\section{Isabelle Périer}

\section{(2) OpenEdition \\ Journals}

Édition électronique

URL : http://journals.openedition.org/tvseries/2188

DOI : 10.4000/tvseries.2188

ISSN : 2266-0909

Éditeur

GRIC - Groupe de recherche Identités et Cultures

Référence électronique

Isabelle Périer, "Penny Dreadful ou comment la littérature peut-elle donner naissance à une série? », TV/Series [En ligne], 12 | 2017, mis en ligne le 20 septembre 2017, consulté le 19 avril 2019. URL:

http://journals.openedition.org/tvseries/2188; DOI : 10.4000/tvseries.2188

Ce document a été généré automatiquement le 19 avril 2019.

\section{cc) (1)}

$T V /$ Series est mis à disposition selon les termes de la licence Creative Commons Attribution - Pas d'Utilisation Commerciale - Pas de Modification 4.0 International. 


\title{
Penny Dreadful ou comment la littérature peut-elle donner naissance à une série?
}

\author{
Isabelle Périer
}

1 Penny Dreadful est une série fantastique (ou d'horreur, ou dramatique, ou encore, selon Wikipedia, un thriller1) proposée par la chaîne américaine payante Showtime. Elle réunit un casting de choix, avec notamment Eva Green et Timothy Dalton, mais est également le fruit d'une collaboration prestigieuse et déjà placée sous le signe de la littérature et du théâtre. En effet, il s'agit d'une création de John Logan, qui est non seulement scénariste ( The Aviator, Gladiator, Skyfall, Sweeney Todd - adaptation filmique réalisée par Tim Burton d'une comédie musicale de Stephen Sondheim, elle-même adaptée... d'un penny dreadful), mais également auteur de pièces de théâtre et de Sam Mendes qui est à la fois producteur et metteur en scène au cinéma (American Beauty, Skyfall) ainsi qu'au théâtre. Le projet était si prometteur que Showtime a immédiatement commandé, dès janvier 2013, la mise en production du projet, sans passer par la préparation préalable d'un pilote. Il est né de ce projet une première saison de huit épisodes d'environ une heure. Une deuxième saison de dix épisodes a été signée en mai 2014 et sera diffusée à partir de mai 2015.

2 Née de la collaboration de deux hommes aux talents variés, alliant cinéma et littérature, Penny Dreadful est une série qui, dès son titre et son origine, se place sous le signe du livre et de la littérature. Le visionnage de la saison 1 confirme cette première impression et ce, de diverse manières. En effet, les références au livre et à la littérature présentes dans la série ne sont pas un simple motif ou un prétexte «littérarisant», mais elles conditionnent toute son esthétique, dans ses dimensions thématique, visuelle, rythmique, temporelle et même métadiscursive. Nous interrogerons ainsi l'articulation entre la littérature comme texte (et comme intertexte, hypotexte, architexte...) et la présence matérielle récurrente du livre en tant qu'objet.

3 Toutefois, une précaution s'impose. Cet article, pour des raisons temporelles, ne concerne que la première saison: notre approche est donc nécessairement monographique et parcellaire et elle pourra être infirmée ou confirmée par les développements futurs de la 
série. L'étude des narrations sérielles est conditionnée par le mode de production de ces dernières et manque parfois du recul nécessaire pour en proposer une analyse globale et définitive. Prenons donc ce qui va suivre comme l'esquisse d'une réflexion à élargir au fur et à mesure que le récit progressera vers son hypothétique fin.

\section{Présence de la littérature dans la série}

\section{Des personnages littéraires célèbres}

4 La première présence évidente de la littérature dans la série Penny Dreadful est l'omniprésence de quatre grandes références littéraires qui constituent les quatre grands fils de la trame de cette première saison de la série. La première est celle de Frankenstein ou le Prométhée moderne de Mary Shelley. En effet, Sir Malcolm et Vanessa Ives sont assistés, dans leur mission, par un certain docteur Frankenstein que l'on découvre, au fil de la saison, hanté par son passé et par la créature étrange à laquelle il a donné naissance. La deuxième référence est celle au Portrait de Dorian Gray d'Oscar Wilde. Les personnages font rapidement la connaissance d'un très charmant jeune homme nommé Dorian Gray qui est un hédoniste impénitent. La troisième est une trame de fond liée au Dracula de Bram Stoker puisque Sir Malcolm a perdu sa fille Mina, tombée sous la coupe d'une créature surnaturelle monstrueuse. La quatrième et dernière référence, plus proche de la culture populaire, du cinéma, et moins évidente, est, selon toute vraisemblance, un loupgarou nommé Ethan Chandler qui pourrait faire référence au film Le Loup-garou de Londres ou bien encore au roman steampunk de Brian Stableford Les Loups Garous de Londres.

5 La présence simultanée et conjointe de ces personnages a de quoi étonner le spectateur. Ces œuvres appartiennent bien à un imaginaire collectif conjoint - celui du roman gothique et du fantastique britannique, ainsi que celui de ses réécritures nombreuses, littéraires ou cinématographiques - mais elles appartiennent à des périodes différentes. Mary Shelley publie son Frankenstein en 1818, Oscar Wilde crée son Dorian Gray en 1890 et Bram Stoker, plus proche d'Oscar Wilde que de Mary Shelley, publie Dracula en 1897. Quant au Loup Garou de Londres, il appartient davantage à une mythologie populaire qu'à une œuvre littéraire fondatrice et, si œuvre fondatrice il y a, elle oscille entre un film datant de 1935 et deux romans parus en 1981 et 1990.

Ces œuvres sont utilisées et réécrites de diverses manières. Dorian Gray est repris en tant que personnage de dandy décadent et en marge de la société. On le reconnaît aux traits caractéristiques que la postérité a gardé de lui ${ }^{2}$ : son attitude de dandy, sa corruption morale et sa perversité, son goût du théâtre et surtout son tableau, que, très habilement, la série ne montre jamais directement ${ }^{3}$. Pourtant, le personnage de Dorian semble figé dans un présent intemporel qui est loin d'être celui du roman d'Oscar Wilde. Le Dracula de Bram Stoker n'est, lui, jamais nommé. Le monstre que Malcolm et Vanessa poursuivent lui ressemble, mais l'intrigue laisse à penser qu'il ne serait pas un monstre unique. Les liens reliant la série au roman originel sont paradoxalement à la fois ténus et omniprésents, car ils constituent un rappel constant de l'hypotexte de Stoker : la fille de Malcolm se nomme Mina, elle est mariée à un certain Jonathan Harker, le monstre est arrivé par un navire infesté et mis en quarantaine dans le port de Londres, il est vénéré par un jeune homme métamorphosé dont la dévotion n'est pas sans rappeler celle de Renfield, et Vanessa rencontre une jeune fille sur un banc qui dit, ironiquement, se nommer Lucy. La série fait également apparaître l'un des personnages principaux du 
Dracula de Stoker : le professeur Van Helsing. Du Frankenstein de Shelley, la série a gardé le monstre originel, en le rebaptisant Caliban, du nom de la créature shakespearienne, sa rage et son éducation livresque, sa laideur et son idée fixe d'obtenir de son créateur une compagne. Toutefois, le récit romanesque dévie de son cours lors de l'analepse narrant la mort de la mère de Frankenstein, qui veut expliquer l'obsession de ce dernier à vaincre la mort. La série transforme également le mythe en montrant une nouvelle naissance, celle de Proteus, qui sera sauvagement démembré par Caliban. De plus, la fin de la première saison voit Frankenstein, touché par la peine du monstre, hésiter à le tuer et changer d'avis afin de lui donner une compagne. Chaque récit originel est repris par fragment et transformé, redoublant ainsi l'intérêt du spectateur pris dans un curieux jeu entre la reconnaissance des mythes littéraires originels et l'étonnement devant la métamorphose que leur fait subir la série.

7 Le plaisir du spectateur naît également de l'entremêlement des différents récits qui finissent par donner naissance à un nouveau récit transfictionnel ${ }^{4}$ : Ethan, le Loup-garou de Londres, est engagé par Malcolm et Vanessa pour pourchasser le ravisseur de Mina, c'est-à-dire Dracula ou du moins le monstre qui en tient lieu. Frankenstein les aide de son savoir scientifique. Il rencontre ainsi Ethan et sa compagne Brona et choisira de prendre le corps de cette dernière pour créer une compagne à Caliban. Celui-ci travaille dans le théâtre où se rencontrent les personnages et qui abrite le vampire détenant Mina. Dorian fait la cour à Vanessa mais croise le chemin de Brona, puis celui d'Ethan avec qui il passera finalement une nuit. Frankenstein se lie avec le Pr. Van Helsing qui sera tué par Caliban, sa créature... Ces allusions littéraires finissent assez naturellement par s'entremêler jusqu'à forger une nouvelle intrigue, propre cette fois-ci à la série, dans un jeu entre reconnaissance, nouveauté et contamination.

\section{D’autres références littéraires en sourdine}

8 La série mobilise également nombre de références littéraires: Wordsworth, Keats, Shelley, Milton, Shakespeare, Wagner, les penny dreadfuls, le Grand Guignol... La liste est longue mais elle peut être classée en trois pôles principaux : la poésie anglaise, le théâtre sous diverses formes (Shakespeare, l'opéra de Wagner, le genre populaire du Grand Guignol), le romanesque surnaturel et horrifique des penny dreadfuls 5 . Cette liste, pour le moins hétéroclite, est également représentative du travail de tissage - ou de métissage entre culture savante et culture populaire que la série s'efforce de mettre en œuvre.

L'omniprésence des poètes anglais s'explique de diverses manières. D'abord, il s'agit d'une référence aux sources ayant nourri l'imaginaire et de la culture britannique: Frankenstein cite un vers de l'Adonais de Shelley ${ }^{6}$ à Van Helsing, qui donne sens à ses paroles à ce moment-là, mais constitue également un clin d'œil littéraire puisque Percy Shelley est l'époux de Mary Shelley. De même, Caliban cite Le Paradis perdu de Milton à Maud afin de qualifier sa condition. Les poètes anglais, et notamment les poètes romantiques, sont également un intertexte extrêmement courant dans la littérature postmoderne du gaslight romance ou du steampunk sur lesquels nous reviendrons.

Shakespeare, quant à lui, semble être une référence à la fois esthétique et métadiscursive, fonctionnant comme une source d'archétypes pour la série, notamment dans le réemploi de ses personnages. Vanessa fait une allusion à la Tempête et au personnage d'Ariel pour son faucon empaillé tandis que Frankenstein nomme son deuxième « monstre » Proteus, en lui faisant choisir un nom au hasard dans son recueil de Shakespeare. De façon 
ironique, son premier monstre est nommé Caliban par Vincent, confirmant ainsi la nature complexe du personnage. Mais on peut également relier la mention de Shakespeare à celle de Wagner et du Grand Guignol. Shakespeare est un dramaturge qui n'a jamais hésité à mélanger les genres et les registres : il peut être considéré comme une figure tutélaire de l'hybridation littéraire (et donc sérielle!). Le Grand Guignol, comme le souligne le personnage de Vincent qui fait allusion à ses réécritures de Shakespeare, est également le lieu de l'hybridation, et peut-être faut-il lire dans ce sens la mention de Wagner (1.4) qui hybride les arts afin de créer un spectacle total.

\section{Des références plus diffuses}

11 La série est enfin un tissu de références littéraires qui fonctionnent davantage sur le mode de l'allusion ou de l'analogie que de la réelle reprise inter- ou hypotextuelle: on peut lier le thème égyptien à toute une littérature gothique et fantastique de l'époque, de Poe (Petite Discussion avec une momie) à Bram Stoker (Le Joyau des sept étoiles) en passant par l'attrait manifesté par Lovecraft pour les civilisations antiques et pour l'Égypte, au travers notamment du personnage de Nyarlatothep, surnommé le Pharaon Noir. On peut s'interroger sur les affinités lointaines mais peut-être bien réelles que peuvent nourrir $L a$ Dame aux Camélias d'Alexandre Dumas fils et le personnage de Brona. Ou encore sur la reprise de la thématique du théâtre comme refuge du monstre inspirée du roman de Gaston Leroux nommé Le Fantôme de l'opéra.

Ces références diffuses font également appel à un substrat de culture pop ou «culture geek» selon les mots de David Peyron ${ }^{7}$. En effet, le groupe de personnages réuni par la série n'est pas sans rappeler le comic transfictionnel nommé La Ligue des Gentlemen extraordinaires d'Alan Moore et Kevin O'Neill, les références à Jack l'éventreur (1.2) éveillent tout un hypotexte allant des nombreux romans reprenant son histoire à la bande dessinée From Hell du même Alan Moore et d'Eddie Campbell qui met bien en valeur les bas-fonds de Londres, l'égyptomanie fin de siècle est un topos des jeux de rôle fantastiques mettant en scène la Grande Bretagne victorienne, la gamme Cthulhu by gaslight en tête. Et donc, bien évidemment, ces références ne cessent de réactiver l'architexte que constituent les genres du steampunk et du gaslight romance. Penny Dreadful pioche ainsi dans un imaginaire de la culture populaire pour mettre en place son univers et son récit.

\section{Une sur-représentation de l'objet-livre}

13 Les références à la littérature se font également grâce à la représentation, voire la surreprésentation, de l'objet-livre dans la série. Celle-ci exhibe son substrat littéraire en mettant le livre au cœur de ses représentations.

Le livre est d'abord présent sous la forme de bibliothèques : la maison de sir Malcolm et Vanessa en abrite une gigantesque, qui est souvent mise en valeur car elle constitue l'arrière-plan de nombreuses scènes. Sir Malcolm possède une autre bibliothèque située dans le bureau qui sert à préparer ses expéditions. Le motif de la bibliothèque est également présent avec le personnage de Caliban, qui possède une petite bibliothèque constituant sa seule richesse. Et c'est aussi un motif discret mais bien réel apparaissant chez l'égyptologue Lyle et chez le Pr. Van Helsing. 

déplace jamais sans ses volumes de Shakespeare et des poètes lyriques romantiques, comme le lui fait remarquer Vanessa (1.2). Il donne un nom à sa deuxième créature, Proteus, grâce à un exemplaire de Shakespeare et comprend que Proteus est un ancien marin, grâce au Moby Dick de Melville. Le livre est également un présent : Caliban offre un exemplaire du Paradis Perdu de Milton à Maud, dans l'espoir de partager quelque chose avec elle, de lui faire comprendre sa condition, et peut-être de la séduire.

16$$
\text { (5) }
$$
(1.5) semblent constituer un véritable roman épistolaire, ce que confirmerait la structure de l'épisode sur lequel nous reviendrons. La carapace de la première créature capturée par Malcolm et Vanessa constitue un exemplaire d'un ouvrage célèbre : le Livre des Morts égyptien, véritable légende archéologique qui a fait couler beaucoup d'encre et défiler beaucoup de pellicule. Enfin, le théâtre de Vincent, le Grand Guignol, peut être considéré, par métonymie, comme une présence indirecte du livre dans la narration.

17 À première vue, ce recensement des références littéraires et livresques de la série laisse à penser qu'elle constitue surtout un mash-up informe où la littérature, ou plutôt des bribes de littérature, seraient davantage un prétexte à développer des histoires surnaturelles en utilisant des personnages connus au lieu d'en inventer de nouveaux. La littérature n'y serait qu'un prétexte servant une forme sérielle fantastique traditionnelle. Mais il n'en est rien : Penny Dreadful, loin de n'être qu'un agrégat informe de références hétéroclites, se constitue en série d'auteur, selon la définition qu'en donne Vincent Colonna dans le deuxième opus de son ouvrage sur les séries, qui unifie les références littéraires de diverses manières, afin de constituer une œuvre esthétique proche d'une construction littéraire et cinématographique.

\section{Penny Dreadful, une série d'auteur littéraire et esthétisante}

\section{Une unité thématique}

18 Penny Dreadful est une série unifiée par quelques grandes thématiques qui permettent de lier les différents références mises en œuvres et de donner une unité à la série. Elles relèvent à la fois du genre gothique et des penny dreadfuls, mais constituent également des objets de pensée contemporains voire intemporels. La première grande thématique est constituée par l'opposition vie/mort qui est rapidement dépassée par l'existence du « demimonde ». Ainsi, il s'agit de vaincre la mort en créant une nouvelle vie pour Caliban, de savoir si Mina est morte ou vivante, de voir Brona se poser de nombreuses questions sur la mort lors de son agonie ou encore de côtoyer Dorian Gray qui ne peut plus mourir, comme on le voit dans la séquence où ses plaies se referment (1.7). Vanessa est souvent projetée dans le «demimonde » et supplie Ethan de lui donner la mort pour ne plus blesser ses compagnons et échapper à ce qui la poursuit. Quant à Ethan, il est entouré par la mort : responsable de massacres aux États-Unis et probablement à Londres, il s'est épris de Brona qui meurt à la fin de cette première saison.

19 Le deuxième fil directeur, lié à cette problématique vie/mort est celle de l'immortalité : cette immortalité est celle d'Amun-Râ, probablement le Maître qui poursuit Vanessa et possède le pouvoir de renaissance et la vie éternelle en se nourrissant d'autres âmes (1.2). 
Mais c'est aussi celle de Caliban qui ne peut plus mourir et constitue, comme l'avait déjà souligné Mary Shelley, une nouvelle espèce. Frankenstein est également celui qui peut donner l'immortalité : la série lui confère la capacité de recommencer l'opération ad libitum. Le monstre qui retient Mina prisonnière semble également doté de vie éternelle, tout comme Dorian Gray. Enfin, Ethan, en tant que loup-garou, n'est absolument pas inquiété par la maladie de Brona, comme s'il était doté d'un organisme à la vitalité surnaturelle.

La dernière grande opposition qui unifie toute la série et ses personnages est celle entre le normal et le monstrueux. Tous les personnages de la série, Brona mise à part - mais elle s'en rend compte, s'en offusque (1.4) et en meurt peut-être pour renaitre sous la forme d'un monstre (1.8) - sont d'une manière ou une autre monstrueux. Frankenstein est un monstre moral, qui veut vaincre la mort, prône des théories positivistes et est esclave de ses recherches qui constituent le centre de sa vie. Il a enfanté un monstre, Caliban, qui, du fait de son physique, se retrouve mis au ban de la société. Le professeur Van Helsing incarne une tentative de vivre normalement lorsqu'il parle de sa femme et de l'amour qu'il lui portait. Il essaie de détourner Frankenstein de la vie anormale et monstrueuse qu'il a lui-même vécue, notamment en tuant de ses mains le monstre qu'était devenu sa femme (1.6). Sir Malcolm est présenté, peu à peu, comme une sorte de monstre moral, obsédé par la quête des sources du Nil et qui aurait sacrifié sa famille, et notamment son fils, à cette obsession (1.7). Dorian Gray est également présenté comme monstrueux à deux titres : il est immortel et amoral. Pire, il vit son amoralité sans même en tirer de plaisir particulier: photos de nu, attirance morbide pour Brona et sa maladie, relation avec Ethan - qu'il a vu avec Brona -, combats de chiens clandestins, orgies... Ethan semble habité par un monstre qu'il ne contrôle pas : on comprend peu à peu que la séquence de départ, qui voit le meurtre sanglant et abominable d'une femme et de sa fille, pourrait bien être de son fait; on apprend qu'il est responsable de massacres dans son Amérique natale; et la fin de la saison le montre en train de se transformer afin d'éliminer les deux agents de Pinkerton venus le ramener à son père - la mise en scène laisse entendre qu'il se livre alors à un massacre de toute l'auberge où il s'était installé pour prendre un verre. Enfin, Vanessa est également un monstre : on comprend qu'elle serait une réincarnation d'Amunet - qui la possède en 1.2 -, une divinité égyptienne elle-même habitée par un monstre. Vanessa ne peut accéder à la normalité que représentent pour elle Mina et Peter : possédée par quelque chose qui la dépasse, elle ne peut vivre une vie normale. Cette plongée dans l'anormalité est narrée dans l'épisode 5 qui constitue une longue analepse montrant le passé de Vanessa et Malcolm. Mais surtout, quelques répliques de la série interrogent sa volonté de redevenir normale : lorsque Dorian et Ethan quittent le théâtre pour " oublier qui ils sont » (1.4), Ethan se préoccupe de Vanessa, laissée derrière eux et Dorian lui rétorque qu'elle ne veut pas oublier qui elle est. De même, la fin de la saison met en scène son tête-à-tête avec un prêtre catholique qui lui parle d'exorcisme mais lui demande, littéralement, si elle désire être normale et échapper au « sacré » et à la "gloire de la souffrance ". La série répond ainsi au goût du Mal et du monstrueux qui caractériserait, selon Vincent Colonna, la série d'auteur après les années 90.

21 Ces thématiques entremêlées permettent d'unifier le mash-up littéraire que semble constituer Penny Dreadful et de donner à la série consistance et cohérence en justifiant son utilisation de la transfictionnalité la plus hétérogène. 


\section{Une unité esthétique}

22 L'unité esthétique de la série est en réalité une trinité. Sa première caractéristique est de relever d'un architexte de type steampunk, ou plutôt du gaslight romance ${ }^{8}$. Ethan mis à part, car il est toujours montré comme un exilé et un marginal, les personnages viennent de Grande Bretagne, ils appartiennent à un hypotexte britannique et sont réunis à Londres qui constitue, l'analepse centrale (1.5) mise à part, l'unité de lieu de la série. Même les références plus exotiques, notamment à l'Égypte, relèvent d'une égyptomanie caractéristique de la Grande Bretagne : le personnage de Malcolm, qui réécrit la légende de Richard Francis Burton - le personnage incarné par Timothy Dalton ressemble de manière troublante aux photographies de Burton - unifie cette référence à l'Égypte et l'incorpore au reste des références britanniques et architextuelles du gaslight romance, qui n'est - au fond - qu'un héritier de la littérature gothique.

La deuxième caractéristique esthétique est annoncée dès le titre de la série: les penny dreadfuls. Cette référence esthétique se prolonge avec celle du Grand Guignol, par l'intermédiaire notamment du théâtre de Vincent dans lequel habitent Caliban et le monstre que cherchent Malcolm et Vanessa. Ce théâtre du Grand Guignol est une allusion transparente à la salle de spectacle parisienne du même nom. Sa caractéristique était de proposer des spectacles à la fois macabres et sanguinolents à l'aide d'effets spéciaux spectaculaires. On en voit un exemple dans l'épisode 4 où Brona et Ethan vont au théâtre et rencontrent Dorian et Vanessa. La pièce représentée, The Transformed Beast, qui est un clin d'œil à la véritable nature d'Ethan, se termine sur la mort sanglante de la belle ingénue, à grand renfort de peinture rouge. Or cette représentation est très proche de certaines scènes où le sang coule abondamment, voire gicle. C'est le cas par exemple de la mort de la mère de Frankenstein qui, au milieu d'une conversation, se sent mal et vomit à longs traits une impressionnante quantité de sang (1.3). Ainsi, le dénouement de The Transformed Beast constitue en quelque sorte une mise en abyme (de théatre dans une série) métadiscursive sur l'esthétique de la série, qui trouve dans cette référence au Grand Guignol et à ses effets sanguinolents une unité de ton.

Toutefois, les effets spéciaux sont finalement assez discrets. Cette caractéristique peut s'expliquer par la nature sérielle de l'œuvre, qui ne peut pas forcément mobiliser les mêmes moyens qu'un film. Toutefois, on sait depuis Game of Thrones (HBO, 2011-) que ces considérations financières n'arrêtent pas toujours les showrunners. On peut noter la présence d'effets spéciaux spectaculaires : par exemple lors de la première descente de Vanessa, Malcolm et Ethan dans l'antre du mal (1.1), ou encore lorsque Ethan se transforme dans le dernier épisode. Toutefois, la plupart du temps, les effets spéciaux sont relativement simples voire sobres : la voix du Maître s'adressant à Vanessa lors de ses étreintes avec Dorian Gray se fait sur le mode du ralenti, de la surimpression et de la voix off travaillée (1.6). Vanessa lévite et tourne sur elle-même au début de sa crise finale (1.6). La scène de la réanimation de Proteus par Frankenstein commence par un éclair, puis la lumière s'éteint, ne dévoilant rien du mystère de la résurrection. L'exorcisme sauvage d'Ethan sur Vanessa joue davantage sur le jeu d'acteurs, les bruitages et le son que sur de réels effets spéciaux. Les effets recherchés sont souvent des effets de lumière ou de son et l'ambiance lourde et surnaturelle surgit bien souvent d'effets de camera où celle-ci rase le sol en glissant ou effectue d'impressionnants travellings arrière (1.7). 
Finalement l'unité esthétique, sinon esthétisante, de la série réside également dans le soin apporté aux images et à la bande sonore ainsi qu'à sa dimension foncièrement visuelle. Cette volonté esthétique est annoncée dès le générique très travaillé qui annonce les grands thèmes et présente les personnages principaux de la série. Elle se prolonge dans toute la série par des cadrages extrêmement précis et signifiants, des plans inattendus et même parfois maniéristes. Cette attention à l'image est doublée d'un grand soin apporté aux bruits et à la musique, particulièrement bien adaptée au sujet, parfois grandiloquente, comme le propos global de la série, mais également parfois effacée pour rendre toute sa place au silence. Car la série contient beaucoup de scènes soit musicales, soit silencieuses. Vincent Colonna soulignait dans son premier ouvrage ${ }^{9}$ le logocentrisme de la série télévisée, qui dit tout sans avoir forcément besoin de montrer. Au contraire, dans son deuxième ouvrage ${ }^{10}$, il souligne que l'une des marques de la série d'auteur consiste, comme dans le cinéma d'auteur, à montrer les choses plus qu'à les dire. Ainsi la série, plutôt que d'expliquer tout à Ethan Chandler - et donc au spectateur - dans le premier épisode, préfère le plonger dans une expédition étrange où tout le mystère du monde invisible est d'un coup dévoilé. Il en va de même lors de la découverte du monde extérieur par Proteus : la musique qui joue en sourdine met en valeur le quasi silence des personnages et l'omniprésence des bruits et des images souligne le choc sensoriel de la créature. Ainsi, Penny Dreadful propose une esthétique marquée par le gaslight romance et le Grand Guignol mais travaillée à l'extrême, dans le sens d'une cinématographisation de la série, tant dans son esthétique globale que dans son rythme même.

\section{Un récit au rythme et à la temporalité particuliers}

Dans l'un de ses articles, Michael Newman montre que les séries de type prime time, qu'on pourrait assimiler aux séries traditionnelles décrites par Vincent Colonna dans son premier ouvrage, possèdent un rythme qui leur est propre, articulant le récit en scènes ou " pulsations ", en épisodes et en arcs narratifs qui s'entremêlent ${ }^{11}$. Or Penny Dreadful, en tant que série d'auteur diffusée sur Showtime - une chaîne payante concurrente de HBO -, ne participe pas de ce rythme particulier.

D'abord, on peine à y retrouver la notion de pulsation. Des scènes très longues alternent avec des rushs très courts, qui viennent ponctuer l'action ou orienter l'attention du spectateur sur un autre arc narratif. On ne peut plus réellement parler de pulsation. Par exemple, l'épisode 2 met en scène une visite de Vanessa et sir Malcolm chez l'égyptologue Lyle. Cette scène peut éventuellement être découpée en sous-scènes, mais son unité de lieu et de personnages ainsi que la continuité de l'enchaînement des scènes, qui ne sont pas interrompues par une pulsation issue d'un autre arc narratif ou tournant autour d'un autre personnage, nous poussent à la considérer comme une unité.

\begin{tabular}{|l|l|}
\hline Minutage & Sous-scènes \\
\hline $25^{\prime} 39$ & Arrivée et accueil de sir Malcolm et Vanessa \\
\hline $27^{\prime} 05$ & Rencontre de Vanessa et Dorian \\
\hline $29^{\prime} 20$ & Début de la séance de spiritisme \\
\hline
\end{tabular}




\begin{tabular}{|l|l|}
\hline $32 ’ 30$ & Possession de Vanessa (1) \\
\hline $366^{\prime} 17$ & Possession de Vanessa (2) \\
\hline 38,24 & Fin de la réception \\
\hline
\end{tabular}
le systématiser comme dans Heroes (NBC, 2006-2010) ou dans Lost (ABC, 2004-2010). Dans Penny Dreadful, certains personnages voient leur passé dévoilé par analepse, comme Frankenstein (1.3), Caliban (1.3), ou encore Vanessa et, dans une moindre mesure, sir Malcolm (1.5). D'autres personnages racontent leur vie à un ou plusieurs autres personnages comme sir Malcolm et sa tragédie familiale, Brona ou Van Helsing. D'autres encore ne disent rien, comme Dorian Gray, ou livrent quelques bribes de passé au cours des dialogues comme Ethan. L'attention du spectateur est donc requise pour reconstituer les pièces d'un puzzle qu'on ne lui livre pas partie par partie, mais dont il doit glaner les pièces $^{12}$. Le plus significatif de ce travail demandé au spectateur réside dans l'évocation d'éléments du passé, en vrac et sans explication, qui ne seront expliqués que plus tard. Par exemple, la scène de possession de Vanessa dévoile de nombreuses informations sur le passé de Malcolm et de sa famille, mais ces informations restent complètement mystérieuses pour le spectateur, provoquant sa curiosité ${ }^{13}$ en soulevant des questions sans y répondre immédiatement.

Du point de vue des arcs narratifs, certains épisodes gardent le principe de leur entremêlement tout en favorisant l'un d'entre eux. Par exemple, l'épisode 1 entremêle l'arc d'Ethan, celui de Vanessa et de Malcolm, sans les distinguer réellement, et celui de Frankenstein. L'arc dominant est celui de Vanessa et Malcolm, car il constitue le socle fédérateur de l'intrigue de la saison, qui s'achève sur leur victoire contre le monstre et sur la mort de Mina, celui autour duquel viennent se greffer les autres arcs narratifs. Au contraire, l'épisode 3 se centre principalement sur les arcs narratifs de Frankenstein et Caliban, dans un principe d'emboîtement analeptique puisque Caliban raconte son histoire au sein de l'arc narratif de Frankenstein, tout en faisant avancer l'intrigue générale avec la nuit passée au zoo. Toutefois, la dominante est parfois telle qu'elle tend à faire disparaître les autres arcs. L'arc narratif principal que constitue la possession de Vanessa conditionne tout l'épisode 7, en lui imposant un rythme faisant alterner crises et pauses. Ces pauses permettent d'une part de mettre brièvement en scène les autres personnages qui, mis sous tension par les événements, se livrent de plus en plus, mais elles sont aussi un moyen de faire redescendre la tension narrative et de mettre en place une série de paliers dans l'horreur de la possession, jusqu'à la résolution finale qui culmine en une acmé remarquable, l'exorcisme sauvage d'Ethan sur Vanessa. Ce jeu sur l'écriture sérielle culmine dans l'épisode 5 , qui est placé à dessein au centre de la série et qui constitue une gigantesque analepse explicative traitée comme un moyen métrage de 55 minutes. Car cet épisode ne repose que sur un seul arc narratif : le passé de Vanessa et de Malcolm et l'explication de leur étrange destin. Ainsi, il tend à se transformer en œuvre unitaire, qui pourrait être regardée telle quelle. Il constitue une longue pause dans 
la temporalité de la série et quasiment une œuvre autonome. Le plan initial montrant Vanessa en train d'écrire une lettre, l'utilisation de la voix-off dans le premier tiers de l'épisode ainsi que le plan final de l'épisode sur son coffre à lettres suggère le rapprochement avec le roman épistolaire et constitue très probablement un clin d'œil métadiscursif au Dracula de Bram Stoker dont l'épisode constitue en quelque sorte une préquelle transfictionnelle.

Cette temporalité particulière est assez sensible au premier visionnage, qui laisse parfois l'impression d'un problème de rythme et provoque de faux souvenirs chez le spectateur qu'un revisionnage attentif stylo à la main vient corriger. Cette impression naît de notre habitude à un certain rythme sériel qui est ici bouleversé, non seulement comme on l'a vu dans ses détails, mais également dans sa répartition à l'échelle de la saison. Car la première saison de Penny Dreadful, comme de plus en plus de saisons de séries d'auteur, à l'instar de True Detective (HBO, 2014-), ne répond pas tant à un rythme spécifiquement sériel qu'aux étapes d'un scénario hollywoodien. Celui-ci est très largement codifié : je m’appuierai sur le modèle en quinze étapes exposé par Blake Snyder ${ }^{14}$.

\begin{tabular}{|c|c|}
\hline Temps forts & Temps forts de Penny Dreadful (S1) \\
\hline $\begin{array}{l}\text { 1) L'image } \\
\text { d'ouverture }\end{array}$ & Scène initiale de massacre (1.1). \\
\hline $\begin{array}{l}\text { 2) L'établissement } \\
\text { de la thématique }\end{array}$ & Descente dans la fumerie d'opium et le « demimonde » (1.1). \\
\hline 3) L'exposition & Explications à Ethan Chandler et Frankenstein (1.1). \\
\hline $\begin{array}{l}\text { 4) Le catalyseur et 5) } \\
\text { L'hésitation }\end{array}$ & $\begin{array}{l}\text { Frankenstein et Ethan hésitent à accepter de travailler avec Vanessa et } \\
\text { Malcolm. Frankenstein est poussé par la curiosité et par les mystères que } \\
\text { lui dévoile Malcolm, Ethan hésite pour Brona dont la rencontre et la } \\
\text { maladie constituent le catalyseur (1.1,2 et } 3 \text { ). }\end{array}$ \\
\hline $\begin{array}{l}\text { 6) Le passage à } \\
\text { l'Acte II }\end{array}$ & $\begin{array}{l}\text { Rencontre de Dorian (1.2), scène de possession (1.2), révélations sur } \\
\text { Amunet et Amun-Râ (1.2), mort de Proteus (1.2) et découverte de Caliban } \\
\text { (1.3), sortie au zoo et capture de Fenton (1.3). }\end{array}$ \\
\hline $\begin{array}{l}\text { 7) L'intrigue } \\
\text { secondaire }\end{array}$ & $\begin{array}{l}\text { Analepses de Frankenstein et de Caliban (E3), découverte du personnage } \\
\text { de Dorian (1.3). }\end{array}$ \\
\hline 8) Humour et jeux & $\begin{array}{l}\text { Détente du théâtre (1.4), soirée et nuit de Dorian et Ethan qui désirent, } \\
\text { pour une nuit «être quelqu'un d'autre » (1.4), parenthèse autour du } \\
\text { passé de Vanessa et Malcolm (jeu narratif) (1.5). }\end{array}$ \\
\hline 9) Le pivot central & Sortie de Vanessa et Dorian, dîner, nuit torride (1.6). \\
\hline $\begin{array}{l}\text { 10) Le méchant se } \\
\text { rapproche }\end{array}$ & $\begin{array}{l}\text { Sortie au port de Malcolm, Ethan et Sembene qui voient brièvement Mina } \\
\text { et le monstre (1.6), relâchement du contrôle de Vanessa et contact avec le } \\
\text { Maître (1.6), Caliban tue Van Helsing (1.6). }\end{array}$ \\
\hline
\end{tabular}




\begin{tabular}{|c|c|}
\hline $\begin{array}{l}\text { 11) Tout est perdu et } \\
\text { 12) La nuit sombre } \\
\text { de l'âme }\end{array}$ & Longue et éreintante possession de Vanessa et quasi-mort (1.7). \\
\hline $\begin{array}{l}\text { 13) Le passage à } \\
\text { l'Acte III }\end{array}$ & Réveil de Vanessa qui a une vision du lieu où se trouve Mina (1.7). \\
\hline 14) Le final & $\begin{array}{l}\text { Rupture entre Vanessa et Dorian, trop dangereux (1.8), mort de Brona et } \\
\text { résolution de Frankenstein de donner une compagne à Caliban (1.8), } \\
\text { Ethan écrasé de chagrin et pourchassé par son passé tue ses poursuivants } \\
\text { (1.8), scène finale dans le théâtre où Malcolm tue le monstre et Mina } \\
(1.8) \text {, il renonce à son voyage en Afrique et choisit Vanessa comme fille } \\
(1.8) \text {. } \\
\text { => Lignes restées ouvertes pour une deuxième saison : Vanessa et Dorian, } \\
\text { rejeté et triste; la résurrection possible de Brona entre les mains de } \\
\text { Frankenstein; Ethan et son passé ; futur de Vanessa, toujours menacée } \\
\text { par le Maître. }\end{array}$ \\
\hline 15) L'image de fin & $\begin{array}{l}\text { Dialogue entre Vanessa, entrée dans l'église qu'elle contemplait dans } \\
\text { l'épisode } 4 \text {, et le prêtre, qui lui demande finalement si elle désire } \\
\text { réellement être normale (1.8). }\end{array}$ \\
\hline
\end{tabular}

Si l'on adopte pour critère scénaristique les quinze étapes que nous venons de mentionner, la saison 1 de Penny Dreadful semble bien s'y conformer, y compris même dans le titre de certains temps forts (« La nuit sombre de l'âme » résume particulièrement bien l'épisode 7 centré sur la possession de Vanessa et son sort tragique).

\section{Une unité métadiscursive}

La dernière unité qui contribue à faire de Penny Dreadful une œuvre sérielle d'auteur est constituée par l'omniprésence du métadiscours qui émaille toute la série.

Le premier aspect métadiscursif à relever est générique. La série, en quelques occasions, fait allusion aux genres littéraires qui l'ont nourrie. Nous avons vu que le titre lui-même et la référence au Grand Guignol est déjà une indication métadiscursive et esthétique. $\mathrm{Ce}$ rapport entre la présence du théâtre et le métadiscours qui s'y attache est multiple: pendant la représentation de The Transformed Beast, l'acteur Vincent déclare: "There cannot be an happy ending ${ }^{15}$ » à son histoire de bête humaine. De plus, on a vu que ce passage met au jour, par une mise en abyme, les codes du Grand Guignol qui sont également les codes esthétiques de la série. Mais les allusions génériques sont aussi littéraires: à Proteus qui découvre le monde extérieur (1.2), Frankenstein montre les lampadaires en mentionnant "the gaslight", rappelant ainsi l'hypotexte de fantasy, héritage du gothique, qui émaille la série. Et Caliban ajoute, un épisode plus tard (1.3): «I am modernity personified. [...] We are men of iron and mechanisation, now. We are steam engines and turbines ${ }^{16}$. $\gg$ La référence à la modernité, en plus d'être un clin d'œil au soustitre de l'œuvre de Mary Shelley, est également une déclaration esthétique évoquant le genre steampunk, sa mécanisation, son goût de l'acier et de la vapeur. Ainsi, dès le début de la série, le métadiscours exhibe ses sources postmodernes. 

relevant de différents genres et dans sa transfictionnalité. Celle-ci est soulignée de manière métadiscursive dans les images et les références mises en œuvre par la série. L'image récurrente de la grande bibliothèque de la maison de Malcolm, derrière laquelle se tient Vanessa, ne serait-elle pas la représentation de ce fonds littéraire qu'utilise la série pour modeler son propre monde? Ce rapprochement métaphorique est très largement suggéré lorsque Van Helsing révèle à Frankenstein l'existence des vampire et l'attire dans sa bibliothèque pour en sortir un penny dreadful qu'il présente comme une source - déformée certes par ce genre de littérature mais bien réelle - d'informations sur l'existence des créatures surnaturelles. Le motif de la bibliothèque est donc bien une représentation métadiscursive du processus créatif de la série. On peut également rapprocher la pièce principale de la demeure de Dorian Gray de ces représentations. Lors de sa visite, Vanessa fait remarquer qu'elle n'abrite que des portraits : cette galerie de portraits peut également être considérée comme une représentation métadiscursive de la transfictionnalité à l'œuvre dans la série ${ }^{17}$.

De plus, le gaslight romance et le steampunk sont par essence des genres transfictionnels et hybrides ${ }^{18}$. Or cette hybridation rejoint le thème du monstre que nous avons déjà évoqué et qui est filé dans toute la série: Frankenstein crée des monstres hybrides mi-humains, mi-immortels, Miss Ives est double, puisqu'elle est possédée par intervalles par des créatures qui la dépassent, Ethan serait un loup-garou, créature hybride par excellence, Mina est à la fois femme et monstre et Malcolm est un personnage mi-fictionnel miréférentiel ressemblant à s'y méprendre à Richard Francis Burton. Et la clé de cette hybridation littéraire est donnée par une image forte : celle du Livre des Morts, trouvé sur le corps de la créature de l'épisode 1 (première hybridation entre livre et créature) et qu'analyse l'égyptologue dans l'épisode 2. Il sursaute devant l'étrangeté de la représentation centrale qui s'y trouve, à savoir Amunet et Amun-Râ : il souligne que cette représentation est impossible, que ces deux personnages ne sont jamais mis ensemble dans la même incantation sous peine de provoquer l'anéantissement des hommes. Si l'on met de côté la dimension prophétique et apocalyptique de cette révélation, on peut lire cette déclaration comme un métadiscours esthétique : la série accole des représentations et des personnages qui ne sont normalement pas mis ensemble mais vont créer quelque chose de nouveau.

La transfictionnalité proliférante de la série Penny Dreadful peut être qualifiée de mash-up, en raison des nombreuses références hétérogènes qu'elle parvient à assembler, telle un monstre digne $\mathrm{du}$ docteur Frankenstein. Toutefois, son esthétique typiquement postmoderne trouve une unité dans une volonté, propre aux auteurs des séries des années 2000, de faire œuvre et de concurrencer la forme longue et légitime du cinéma. En effet, Penny Dreadful constitue une œuvre à part non seulement dans son unité thématique - la représentation du Mal, comme l'a souligné Vincent Colonna ${ }^{19}$ - mais également dans une unité esthétique, narrative et métadiscursive qui en font un objet fascinant à visionner et à étudier. 


\section{BIBLIOGRAPHIE}

BARONI Raphaël, La Tension narrative. Suspense, curiosité et surprise, Paris, Seuil, 2007.

colonna Vincent, L'Art des séries télé 1. L'Appel du happy end, Paris, Payot, Petite Bibliothèque, (2010) 2015.

colonna Vincent, L'Art des séries télé 2. L'Adieu à la morale, Paris, Payot, 2010.

http://sf-encyclopedia.uk/fe.php?nm=gaslight_romance, consulté le 29 janvier 2016.

http://www.bl.uk/romantics-and-victorians/articles/penny-dreadfuls, consulté le 29 janvier 2016.

NEWMAn Michael Z., « From Beats to Arcs: Toward a Poetics of Television Narrative », The Velvet

Light Trap, $\mathrm{N}^{\circ}$ 58, 2006.

PÉRIER Isabelle, «Cette Chimère qu'est le steampunk... », communication pour le colloque Au-delà des Frontières. Hybridation des formes et des genres dans les littératures de l'imaginaire contemporaines ( $\mathrm{XX}^{e}-\mathrm{XXI}^{e}$ siècles) organisé les 15,16 et 17 octobre 2014 par les UR CLARE et TELEM à l'Université Bordeaux-Montaigne (à paraître).

PEYRON David, Culture geek, France, FYP éditions, 2013.

SAINT-GELAIS Richard, Fictions transfuges. La transfictionnalité et ses enjeux, Paris, Seuil, Poétique, 2011.

SNYDER Blake, Les Règles élémentaires pour l'écriture d'un scénario, trad. Brigitte Gauthier, Paris, Éditions Dixit, 2007.

\section{NOTES}

1. https://fr.wikipedia.org/wiki/Penny_Dreadful_(s\%C3\%A9rie_t\%C3\%A9l\%C3\%A9vis\%C3\%A9e), consulté le 29 janvier 2016. Ce flou générique est en soi un indice de l'hétérogénéité des sources et du propos de la série.

2. Vincent Colonna parlerait de "gimmicks" (L'Art des séries télé 1. L'Appel du happy end, Paris, Payot, Petite Bibliothèque, (2010) 2015, p. 152-153).

3. L'épisode 8 de la deuxième saison (« Memento mori ») offre enfin une visualisation du portrait.

4. Richard Saint-Gelais, Fictions transfuges. La transfictionnalité et ses enjeux, Paris, Seuil, Poétique, 2011.

5. http://www.bl.uk/romantics-and-victorians/articles/penny-dreadfuls, consulté le 29 janvier 2016.

6. Rappelons que l'Adonais de Shelley est une élégie pastorale écrite par Percy Shelley en 1821 pour la mort de John Keats.

7. David Peyron, Culture geek, France, FYP éditions, 2013.

8. http://sf-encyclopedia.uk/fe.php?nm=gaslight_romance, consulté le 29 janvier 2016.

9. Colonna, L'art des séries télé 1., op. cit.

10. Vincent Colonna, L'Art des séries télé 2. L'Adieu à la morale, Paris, Payot, 2010. 
11. Michael Z. Newman, «Fram Beats to Arcs: Toward a Poetics of Television Narrative », The Velvet Light Trap, $\mathrm{N}^{\circ}$ 58, 2006, p. 16-28.

12. La deuxième saison met en scène ce processus, avec l'idée du texte multilingue à réassembler, qui constitue un autre procédé métadiscursif.

13. Raphaël Baroni, La Tension narrative. Suspense, curiosité et surprise, Paris, Seuil, 2007.

14. Blake Snyder, Les Règles élémentaires pour l'écriture d'un scénario, trad. Brigitte Gauthier, Paris, Éditions Dixit, 2007, p. 83-100.

15. «Il ne peut y avoir de fin heureuse » (ma traduction).

16. «Je suis la modernité personnifiée. [...] Nous sommes désormais des hommes nés de l'acier et de la mécanisation. Nous sommes des moteurs à vapeur et des turbines. » (ma traduction)

17. Se référer à l'article de Xavier Giudicelli, «Des penny dreadfuls à Penny Dreadful (2014): hybridité générique et fantaisie néo-victorienne », Fantaisie et arts visuels, dir. Françoise Heitz et Emmenuel Le Vagueresse, Reims, Épure, 2015, p. 15-35.

18. Isabelle Périer, "Cette Chimère qu'est le steampunk... », communication pour le colloque Audelà des Frontières. Hybridation des formes et des genres dans les littératures de l'imaginaire contemporaines (XXe-XXIe siècles) organisé les 15, 16 et 17 octobre 2014 par les UR CLARE et TELEM à l'Université Bordeaux-Montaigne (à paraître).

19. Colonna, L'Art des séries télé 2., op. cit.

\section{RÉSUMÉS}

Penny Dreadful est presque un cas d'école pour qui se propose d'analyser les rapports entre les séries télévisées et la littérature : de l'intertextualité à la transfictionnalité, de la citation à l'allusion, de la littérature à l'objet-livre, elle brasse une quantité incroyable de références explicites ou implicites afin de donner naissance à un monde vacillant entre fantastique et horreur. C'est cette richesse que nous nous proposons d'étudier.

L'intrigue et les personnages de la série sont édifiés sur une intertextualité affichée, qui brasse le Dracula de Bram Stoker, le Portrait de Dorian Gray d'Oscar Wilde et le Frankenstein de Mary Shelley. Cette simple énumération constitue un indice du traitement que la série leur réserve : elle s'en sert, plus qu'elle ne les sert, pour donner naissance à un univers transfictionnel où le spectateur doit naviguer entre le plaisir de la nouveauté et celui de la reconnaissance. Mais la série s'appuie également sur nombre de références littéraires hétérogènes, de Shakespeare au Grand Guignol, en passant par les poètes romantiques anglais ou le genre du " penny dreadful » qui lui permettent de tenir un métadiscours sur sa propre esthétique. Plus généralement encore, cette série est un tissu de réminiscences littéraires, alliant culture légitime (égyptomanie et spiritisme, chez Poe par exemple) et culture populaire (comics, jeux de rôle, culture victorienne de type steampunk...). Or, sur ce fond de références hétérogènes, se greffe une surreprésentation de l'objet-livre : cadeaux, bibliothèques, lettres, livre des morts... Au point qu'on pourra se demander en quoi ces différentes représentations du littéraire viennent appuyer le métadiscours produit par les références intertextuelles de la série.

Penny Dreadful is practically a case study for the analysis of the relationship between TV and literature: from intertextuality to transaction, from quotes to allusions, from literature to the book as object, the series includes an astounding number of references (both explicit and implicit) in order to create a world somewhere between horror and the supernatural. It is this 
rich tapestry that I intend to examine.

The story and its characters are openly intertextual, using Bram Stoker's Dracula, Oscar Wilde's Picture of Dorian Gray, and Mary Shelley's Frankenstein. This simple list gives an idea of the treatment the characters receive: they are tools to enter a transactional universe where the viewer must navigate between the pleasure of novelty and that of recognition. But the series also relies on a number of diverse literary references, from Shakespeare to the Grand Guignol Theatre, with the Romantic poets and the "penny dreadful" genre in-between - the latter of these allows the series to maintain a metafictional discourse on its own aesthetics. More generally, the series is a tapestry of literary memories, merging "legitimate" culture (Egyptomania and spiritism, as in Poe's work for example) and popular culture (comics, role-playing games, steampunk Victorianism). However beyond this background array of references, the series displays a fascination for the book as object, which appears as a gift, in libraries, in letters, as a "Book of the Dead" - to the extent that one could wonder how these different representations of literature participate in a metanarrative produced by the series' intertextual references.

\section{INDEX}

Mots-clés : Penny Dreadful, adaptation, transfiction, intermédialité

Keywords : Penny Dreadful, adaptation, transfiction, intermediality

\section{AUTEUR}

\section{ISABELLE PÉRIER}

Ancienne élève de l'ENS de Lyon, agrégée de Lettres classiques et licenciée en Histoire de l'art, Isabelle Périer enseigne aujourd'hui dans un lycée d'Argenteuil. Rattachée au laboratoire du CRI de Grenoble-Stendhal 3, elle est docteur en Littérature comparée et a orienté ses recherches vers la science-fiction et plus généralement les cultures de l'imaginaire. Elle s'intéresse aux narrations sérielles et à la transmédialité, et donc aux séries télévisées («The Tudors ou quand l'histoire se répète », « Destin, Providence et temps cyclique. Quelle liberté pour les personnages du Battlestar Galactica? », « Le conte merveilleux et ses réécritures contemporaines : Grimm et Once upon a time, deux stratégies narratives concurrentes »).

Isabelle Périer has degrees in Classical Studies and Art History, as well as a PhD in Comparative Literature, and teaches in a high school, doing research for the CRI research lab at the University of Grenoble-Stendhal 3. Her research interests include science fiction and fantasy, and she specialises in analysis of serial narratives and transmediality - particularly television series ("The Tudors ou quand l'histoire se répète », « Destin, Providence et temps cyclique. Quelle liberté pour les personnages du Battlestar Galactica? ", « Le conte merveilleux et ses réécritures contemporaines : Grimm et Once upon a time, deux stratégies narratives concurrentes »). 